\title{
Circuit
}

Musiques contemporaines

\section{Un état des lieux, vivifiant}

\section{James Giroudon}

Volume 1, numéro 2, 1990

Montréal musiques actuelles

URI : https://id.erudit.org/iderudit/902012ar

DOI : https://doi.org/10.7202/902012ar

Aller au sommaire du numéro

Éditeur(s)

Les Presses de l'Université de Montréal

ISSN

1183-1693 (imprimé)

1488-9692 (numérique)

Découvrir la revue

Citer cet article

Giroudon, J. (1990). Un état des lieux, vivifiant. Circuit, 1(2), 9-18.

https://doi.org/10.7202/902012ar

Ce document est protégé par la loi sur le droit d'auteur. L'utilisation des services d'Érudit (y compris la reproduction) est assujettie à sa politique d'utilisation que vous pouvez consulter en ligne.

https://apropos.erudit.org/fr/usagers/politique-dutilisation/
Cet article est diffusé et préservé par Érudit.

Érudit est un consortium interuniversitaire sans but lucratif composé de l'Université de Montréal, l'Université Laval et l'Université du Québec à Montréal. Il a pour mission la promotion et la valorisation de la recherche. https://www.erudit.org/fr/ 


\section{Un état des lieux, vivifiant James Giroudon}

Pour New Music America, rendre compte des musiques actuelles, à travers ses multiples tendances, ce n'est pas seulement présenter, pendant une dizaine de jours, des œuvres, des artistes, mais davantage communiquer un état de la création musicale aujourd'hui, en montrant sur quelles transversales elles se cherchent et se construisent. La notion de «musiques actuelles» ne renvoie pas à une esthétique particulière. C'est, au contraire, à la croisée des esthétiques musicales les plus diverses qu'elles se créent. En ce sens, seule la confrontation la plus large, privilégiant l'innovation musicale, et donc pas uniquement un rassemblement de "valeurs sûres" installées à l'intérieur d'un courant musical, est susceptible de révéler les démarches les plus originales et, surtout, de donner un sens à la notion même de "musiques actuelles». La ligne d'horizon est fluctuante, et c'est bien dans l'interaction des œuvres, autant que dans les œuvres ellesmêmes, que se perçoit l'ensemble de la programmation du festival.

Projet réconfortant, énergique, en rupture avec le conformisme des lieux spécialisés en musique contemporaine focalisés autour de chapelles. Projet courageux aussi, puisqu'il s'agit de faire circuler de larges publics dans ce paysage musical tout en mouvements et circonvolutions. Pari réussi, si l'on en juge par la fréquentation des concerts dans les principaux lieux: le Spectrum, le cabaret des Foufounes électriques et la Maison de la culture Frontenac.

New Music America, c'est donc une volonté de procéder à un état des lieux autour des formes les plus variées de l'expression musicale actuelle en Amérique du Nord et de montrer qu'à travers des lignes de forces, en attraction, en complémentarité, en opposition, existe un champ musical présent ou à venir. Même si le tableau est brossé à grands traits, même si l'on peut déplorer certaines absences ou discuter le niveau de représentativité de tel ou tel courant, l'ensemble est passionnant par l'accumulation des prestations, une soixantaine de concerts et performances, de nombreuses créations : un rassemblement tout à fait unique de musiciens sur une durée relativement courte. II sera plus difficile de discerner si, de cette densité, de ces fluctuations, naît la quête d'un nouveau langage, ou si émergent seulement des personnalités, des œuvres spécifiques. 
Compte tenu des conditions quelquefois délicates des concerts (horaires, salles, sonorisation...), un tel programme ne se réalise pas sans risque: certaines prestations s'accommodent fort bien de ce désordre musical, d'autres en sortent davantage meurtries (c'est le cas de la musique électroacoustique); d'autres encore se trouveront mal à l'aise dans ce grand chahut et sembleront se mettre à l'écart de la mêlée: le concert d'ouverture de l'Orchestre Métropolitain ou le concert de la Société de musique contemporaine du Québec, conviant le public, un autre public, au théâtre Maisonneuve et à la salle de l'université McGill. Bref, tout état des lieux ne saurait être exhaustif et ne saurait convenir idéalement à toutes les formes musicales. Il se bâtit sur un certain nombre de contraintes matérielles et de choix et est donc contestable. Mais la photo est suffisamment contrastée, tonique, pour rendre ce parcours musical toujours excitant. L'éclectisme des concerts, la volonté de faire découvrir de nouveaux compositeurs ou d'opérer de fructueuses rencontres musicales provoquent parmi le public une grande disponibilité d'écoute. II faudrait souhaiter à la musique contemporaine de communiquer plus souvent cette sensation.

\section{Un nouveau territoire musical?}

Mais une fois la fête finie, que subsiste-t-il de ce rassemblement? Si le champ des musiques actuelles se définit d'abord par une accumulation d'œuvres, que reste-t-il de chaque musique en particulier? S'il est pertinent de se situer dans ce cadre, s'agit-il d'une véritable synergie entre des pratiques musicales ou d'emprunts stylistiques? S'agit-il de préliminaires, à consonances expérimentales et minimalistes, qui ne permettront à un compositeur de s'affirmer qu'en s'en libérant? En continuant à se définir dans ce contexte, le musicien ne risque-t-il pas de s'épuiser dans un bricolage instrumental et technologique sans fin ou d'être gagné par l'éclectisme, la complaisance? C'est précisément en échappant à ces bornes apparemment contradictoires, mais pourtant délimitant un même tracé, que sont apparus les moments les plus forts du festival. À ces questions, sans réponse, se mêleront, dans ce carnet de notes d'un auditeur plutôt assidu, quelques commentaires sur le sonore, un aspect qui a semblé souvent être trop séparé du musical. 


\section{Le concert, convivial...}

Plus qu'en toute autre circonstance, les conditions des concerts de New Music America créent des situations musicales spécifiques où le feeling du lieu devient un des vecteurs importants pour l'écoute. Le Spectrum et les Foufounes électriques donnent le ton. On se surprend à observer dans d'autres salles, plus conventionnelles dans les rapports scène/public, le rituel bien étrange du concert dans une ambiance feutrée. On ne peut qu'applaudir à cette inversion des normes. L'atmosphère extrêmement conviviale qui imprègne la scène et le public est la première découverte importante du festival. Un cadre trop rigide des concerts, comme nous le connaissons habituellement, rend l'ennui musical tout à fait insupportable. Au contraire, les configurations du Spectrum et des Foufounes, music-hall, boîte branchée, permettent de créer des espaces de proximité et de prendre aisément des distances avec la scène: c'est rendre à la musique sa fonction première de rencontre, et aussi de plaisir.

\section{... au détriment du son}

Sensation contraire lorsque l'atmosphère du lieu génère ses propres nuisances: du détail anecdotique et répétitif de la caisse enregistreuse au brouhaha quelquefois infernal du bar des Foufounes. La musique se partage, elle est aussi un "donné à entendre". La convivialité devient alors privilégiée, aux dépens des conditions de restitution du son dans l'espace. Cette remarque n'est pas seulement d'ordre technique, elle caractérise une démarche, une façon de faire, qui persiste au sein de plusieurs courants musicaux: I'insouciance du sonore est aussi un choix délibéré. On déplore que ce type de norme puisse s'imposer pour l'ensemble des musiques sonorisées. Le niveau en décibels ne règle pas tout, il n'exclut pas la clarté du son: le concert de Test Department en est un bon exemple.

Mais au fil des programmes qui se succèdent, on perçoit vite une même impression sonore. Seule, parmi les lieux les plus fréquentés, la Maison de la culture Frontenac, dans une atmosphère plus soff, offrira une sonorisation toujours correcte: bonne acoustique de la salle, qualité de l'amplification, un ensemble de conditions plus adéquates pour les concerts nécessitant une diffusion par haut-parleurs. Au contraire, le son très "épais", toujours en façade, sans aucune fantaisie de spatialisation, du Spectrum et le son particulièrement déplorable des "Foufounes» installent une monotonie musicale et dessert particulièrement toutes les musiques 
écrites. On peut penser, également, que plusieurs formations n'ont pas bénéficié d'un temps de réglage suffisant de la sonorisation. Le Spectrum a présenté, de ce point de vue, des prestations tout à fait inégales: certains concerts, comme la création de LaMonte Young, ont bénéficié d'une belle présence du son.

Cette revendication du «sonore» pour installer le «musical» ne concerne pas uniquement New Music America, mais elle s'est posée dans ce festival avec beaucoup d'acuité. Paradoxalement, la préoccupation du son est encore une attitude inhabituelle pour la majorité des interprètes : la sonorisation ne serait qu'un problème technique, inintéressant et tout à fait distinct de la musique. Si l'on peut défendre le parti pris systématique de diffusion par haut-parleurs adopté au festival de Montréal, on regrette, pour la musique, que ce choix n'intervienne pas de façon cohérente avec l'instrumentation et l'écriture: absence de plans entre les instruments, effet de sonorisation en bloc, réglages défectueux du son...

Ces regrets amènent à se poser deux types de questions: ce que l'on gagne en convivialité des lieux, comment ne pas le perdre en qualité d'écoute? Comment opérer ces confrontations musicales tout en préservant le caractère propre de chaque œuvre? Certaines démarches musicales à teneur plus expérimentale "jouent» avec les nuisances ambiantes et se servent d'un "gros son" pour appuyer leur esthétique. (Mais ces mêmes musiques alternatives n'auraient-elles rien à gagner en essayant et en exigeant, elles aussi, de bonnes conditions de diffusion?) Cela devient un problème de gestion de salles et d'équipements techniques, à condition que cet aspect ait été pris en considération dans ses multiples rapports aux contenus musicaux.

Encore un regret, celui de n'avoir pas trouvé, auprès de ceux qui peuvent apporter par leur expérience unique en diffusion du son - les créateurs de musique électroacoustique - des démonstrations convaincantes au cours des concerts de la salle Les Loges. Les conditions très rustiques de cette salle ne s'y prêtaient pas.

\section{Une nouvelle expérimentation musicale?}

New Music America à Montréal, c'est d'abord la découverte de multiples facettes de la scène alternative. La première impression est d'entendre une musique qui échappe à tous les classements. De nombreuses esthétiques et des genres s'entrechoquent, se croisent: bruitismes, poèmes sonores, musiques concrètes, électroacoustiques, musique minimaliste, répétitive, jazz, art-rock... chez qui on discernera quelques attentions particulières 
pour l'un ou l'autre franc-tireur ou compositeur atypique de la musique sérieuse de notre siècle. Chacun ou chaque formation s'approprie une partie de ce territoire sous la forme d'un patchwork souvent très coloré.

La "création d'une nouvelle alternative musicale» (Anthony Coleman), à partir de greffes "des éléments musicaux d'une culture (culture balkane) à des éléments d'une autre" définit bien les termes de la recherche de nouvelles géographies musicales, et on prend la mesure de l'ambition. Musiques sous influences, ces musiques improvisées, aux tendances innovatrices, procèdent le plus souvent par mélange des genres, compilations, et n'échappent pas toujours, notamment par leurs propos systématiques, aux conformismes qu'elles affirment rejeter.

Mais lorsque le choc se produit, et il s'est produit à plusieurs reprises, l'émotion musicale est très forte, car dans ce cas, la personnalité, le degré d'expression du musicien est intense. Il faudrait citer le concert de Gordon Monahan, le quartet de Fred Frith, les performances vocales et scéniques de Direct Sound, de David Moss et Carlos Santos, tous deux très impressionnants d'authenticité au cours de solos et de duos vocaux spectaculaires, le groupe Challenge one avec notamment le percussionniste étonnant de précision et d'énergie William Winant, en dialogue avec Anthony Braxton. L'invention musicale est soutenue par un engagement physique total. L'interprète et le compositeur se confondent bien souvent dans un corps à corps spectaculaire. Un peu à l'écart de cette scène, l'itinéraire du violoncelliste Claude Lamothe est tout à fait exemplaire, et ses "manigances» en disent long sur sa disponibilité vis-à-vis de la musique nouvelle: une improvisation se développant à partir de différentes granulations de matériaux de violoncelle et organisée autour de quelques rappels de thèmes et de rythmes. Une place particulière aussi pour Jim Hi Kim dans son improvisation sur un impressionnant instrument coréen, le komungo.

\section{Interprètes, performers, compositeurs?}

L'auditeur vibre en relation intense avec le musicien en spectacle sur scène. Ce sont bien les interprètes qui sont les phares du festival. Ils créent autour d'eux un répertoire original. Confirmation d'un haut niveau de formation, comme pour le quatuor Kronos, associé à un grand souci d'expérimentation et qui représente bien, à travers les œuvres qu'il suscite, un melting-pot de différentes cultures musicales. La pièce d'Istvan Marta, Doom a sigh, en s'inspirant d'un chant de tradition moldave, en est un exemple. Cette formation symbolise ce souci de diversité musicale, de confrontation des styles, sans en exclure l'emprunt. Mais l'écueil d'une musique composite, résistant mal à l'éclectisme auquel elle se soumet, 
ne peut être évité, même par une formation si talentueuse. John Oswald, connu pour son travail de collages sur bande magnétique, proposa, avec Spectre, une pièce mixte (genre plutôt peu répandu à New Music America) d'une grande justesse et homogénéité dans les rapports bande/ instruments, et où la partition semble avoir été construite à partir des qualités du matériau enregistré, ce qui caractérise un travail d'écriture et d'écoute proche de la musique électroacoustique.

Qualité du Nouvel Ensemble Moderne que dirige Lorraine Vaillancourt, notamment à travers l'exactitude de l'interprétation des pièces de Steve Reich et de Stephen Montague, dans un programme qui, tout en restant très accessible, explore aussi d'autres possibilités musicales avec des compositeurs comme Denys Bouliane et Julia Wolfe. On regrettera la diffusion catastrophique de Comme un silène entr'ouvert (Bouliane) qui n'a permis de n'appréhender que partiellement l'œuvre dans ses rapports entre les ensembles musicaux imaginaires sur bande et les trios instrumentaux sur scène. La musicalité de The Vermeer Room (Wolfe) surprend par son caractère à la fois calme et étrange: il en reste le souvenir de blocs sonores traversés de lumière.

Émouvante prestation de la Société de musique contemporaine du Québec avec la très belle œuvre de Claude Vivier, Trois airs pour un opéra imaginaire, interprétée par la soprano Marie-Danielle Parent: musique au caractère dramatique, riche en textures sonores, autonome dans sa forme, où les références ne sont qu'accessoires, où il ne semble demeurer que I'essentiel, comme dans La Question sans réponse de Charles Ives. Beau début et splendide fin de concert sous la direction de Walter Boudreau. Margareth Leng Tan offrit également un moment de grâce au cours de ce festival tonitruant, notamment à travers les pièces de Someï Satoh et de Toschi Ichiyanagi.

À l'engagement de l'interprète et à sa forte présence sur scène - il "porte» véritablement la musique qu'il joue et dont il est quelquefois l'auteur - on prend aussi plaisir à découvrir une grande liberté d'invention de formations instrumentales et vocales, quelquefois inattendues, autour d'une lutherie apparemment rudimentaire (daxophone de Hans Reichel, par exemple): cela montre bien le goût et la disponibilité de ces musiciens pour les aventures les plus diverses.

\section{Musiques minimales, répétitives, toujours}

Hors du débridement sonore de la musique improvisée, les voies de l'écriture musicale semblent étroites, car les musiques répétitives et mini- 
malistes imposent leurs contraintes en agissant comme modèles. On retiendra, parmi les créations du festival, les pièces de Terry Riley (Cactus Rosary par Arraymusic) et de LaMonte Young (Kronos Quartet) qui continuent d'innover en ce domaine, c'est-à-dire de surprendre l'auditeur et de traduire un sentiment profond. LaMonte Young rappelle, dans les notes du programme, qu'il s'agit en fait d'une œuvre complètement écrite, peut-être plus aboutie que des travaux précédents fondés sur une notation algorithmique. Le message est passé: Chronos Kristalla, moment fort du festival, suspendit le temps pour un très large public qui retint sa respiration pendant l'heure et demie que dure cette œuvre strictement minimale.

Performance des interprètes du Kronos Quartet, mais aussi démarche exemplaire du compositeur dans sa plus grande simplicité. La peinture ne s'est pas arrêtée après le carré blanc sur fond blanc de Malevitch, et sans pour autant l'oublier, elle a continué de se diversifier. Comment gérer dans les musiques actuelles I'héritage du minimalisme, du répétitif? On risque la décoloration musicale. Ces esthétiques côtoient les démarches sonores les plus débridées, rock, post-free... et envahissent, malgré tout, l'ensemble de la scène musicale. Des compositeurs comme Satoh, Kucharzyk savent entreprendre de nouvelles démarches musicales. De tout cela, il ressort plus généralement des musiques plaisantes, parfois talentueuses, sans surprise, mais souvent guettées par l'ennui de la répétition.

\section{Et du côté des nouvelles technologies? L'espace du son?}

Dans cette mouvance des musiques actuelles, et dans cette quête d'un renouvellement de l'écriture, on peut déplorer que l'itinéraire de la musique instrumentale soit relativement à l'écart du champ des nouvelles lutheries et technologies de studio. II y a des exceptions, souvent heureuses, qui permettraient d'espérer un apport positif, indispensable, tant sur le plan des matériaux sonores que du point de vue formel. Les techniques de l'électroacoustique n'ont fait encore qu'effleurer la vie musicale. Pourtant la musique sur bande est extrêmement bien représentée, notamment au Québec, à travers des compositeurs comme Francis Dhomont, Christian Calon, Robert Normandeau, Yves Daoust, pour n'en citer que quelques-uns, et aussi par Paul Dolden résidant à Vancouver, avec la pièce Below the Walls of Jericho basée sur un processus accumulatif très maîtrisé (avec une diffusion en façade).

On regrettera d'autant plus la place modeste accordée à la musique sur bande qu'elle avait à montrer beaucoup plus qu'elle n'a pu le faire 
On regrettera d'autant plus la place modeste accordée à la musique sur bande qu'elle avait à montrer beaucoup plus qu'elle n'a pu le faire pendant ce festival. L'enjeu est de taille, car ces compositeurs, à travers des œuvres tout à fait reconnues et diffusées largement à l'étranger, peuvent contribuer à montrer de nouvelles voies et faire partager leurs expériences du sonore.

Par ailleurs, à vouloir faire trop, on atteint le résultat inverse : les concerts des Loges, dans des conditions inadaptées de lieu et d'horaire, semblent avoir souffert de l'excès d'œuvres; une sélection plus sévère aurait considérablement gagné en qualité et crédibilité. Le problème grave de la musique électroacoustique, c'est qu'il y a inflation de compositeurs et d'œuvres, pour un public qui ne connaît pas la même courbe ascendante. Quelques concerts, un seul concert même, dans de bonnes conditions techniques et avec un choix d'œuvres fortes, convaincrait davantage qu'un panorama fastidieux d'enregistrements sur bande (qualificatif choisi à dessein, compte tenu que, trop souvent, il s'agissait d'enregistrements témoins, et non de travaux d'écriture du sonore). De plus, des concerts trop confidentiels entérinent l'idée qu'il ne s'agit là que d'une musique destinée à quelques spécialistes.

On aurait aimé entendre, dans ce cadre, des musiques mixtes des membres de l'ACREQ (Association pour la création et la recherche électroacoustique du Québec), uniquement représentées par les musiques sur bande. Seul le lancement du disque consacré aux électroclips a su briser, par son impact, cette langueur: I'idée de Jean-François Denis et de Claude Schryer, de proposer à une vingtaine de compositeurs de tenir et de resserrer un propos musical sur des formes brèves a abouti à une production originale de miniatures dont on peut espérer une diffusion large, à l'occasion de divers événements, et surtout une insertion dans des programmes radiophoniques.

Une nouvelle génération de compositeurs a entrepris de travailler dans le sens de rencontres entre l'instrumental et les nouvelles technologies: création par l'ensemble Arraymusic de US and THEM de Henry Kucharzyk (mélange d'instruments acoustiques et de dispositifs Midi), dispositifs interactifs de Don Ritter et David Rokeby (interaction vidéo-mouvementmusique), musique mixte de Denys Bouliane (par le Nouvel Ensemble Moderne), de John Oswald (par le quatuor Kronos), musique interactive de Daniel Scheidt (interprétée par le percussionniste Trevor Tureski). Citons encore le groupe Tuyo qui sait explorer avec subtilité l'espace musical: profondeur des plans, proximité de certaines sonorités autour d'une étrange machinerie de tuyaux. Dans ces musiques actuelles, où tout se lit le plus souvent en premier plan, cette architecture du son apporte un souffle vivifiant. 
Dans les festivals de musique contemporaine, le mélange des genres et des styles est généralement dosé avec beaucoup de circonspection. On sacrifie, généralement, à la modernité en proposant quelques programmations ouvertes à des musiciens ne faisant pas partie du sérail, bien que quelques structures d'accueil et festivals adoptent aujourd'hui des positions plus avancées en ce domaine. Mais en se posant la question de savoir comment pourrait être reçu New Music America en France et dans d'autres pays européens, on ne peut s'empêcher de considérer la musique contemporaine "sérieuse» comme un modèle encore coercitif qui, à l'inverse de l'ensemble des «musiques actuelles», prône un état discontinu de la forme, une certaine complexité d'écriture. Cette musique pèche par excès d'autonomie et exclut bien souvent tout recours à l'émotion. Dans ce sens, le déferlement sonore de New Music America est tout à fait salutaire par les chocs qu'il produit, sans que l'on puisse être toutefois convaincu que le terme «musiques actuelles» ne définit pas, aujourd'hui, autre chose que le regroupement, en un lieu, de diverses formes musicales. 


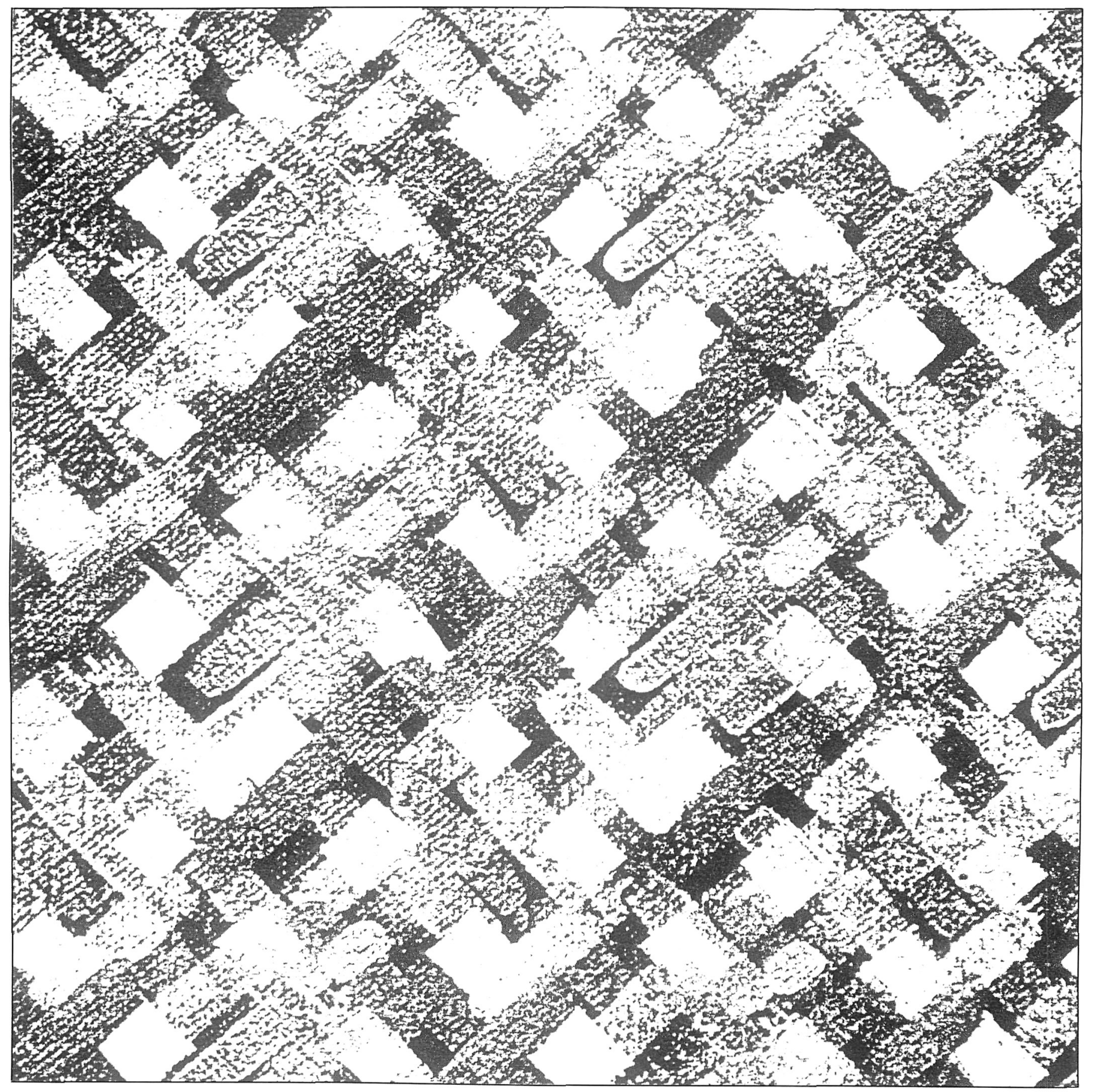

